\title{
Neuroinflammation in neuronal degeneration and repair
}

\author{
L Berliocchi ${ }^{1,2,3}$, MT Corasaniti ${ }^{2,4}$, G Bagetta $^{\star, 1,5}$ and SA Lipton ${ }^{6}$ \\ Cell Death and Differentiation (2007) 14, 883-884. doi:10.1038/sj.cdd.4402097; published online 26 January 2007
}

Workshop on Apoptosis in Biology and Medicine, Parghelia (VV) Italy, 13-16 September 2006.

The IXth workshop on Apoptosis in Biology and Medicine brought together top international scientists to discuss current knowledge and views on the mechanisms of neuroinflammation, on the role of neuroinflammation in the context of different neurodegenerative diseases (i.e., Alzheimer's disease (AD), Parkinson's disease (PD), prion disease, multiple sclerosis, and HIV-associated dementia) and on the potential approaches for future therapeutic strategies. Also, the venue provided a unique opportunity for stimulating discussions particularly for young scientists, postdocs and PhD students, who presented their results in the evening poster sessions. This and previous workshops were organized within the PhD program of the University of Calabria in collaboration with the Universities of Catanzaro and Roma Tor Vergata, and was held in Porto Pirgos in Parghelia (VV, Italy).

In the opening lecture, Stuart Lipton (La Jolla, USA) presented an overview on the role of inflammatory mediators such as nitric oxide (NO) and reactive oxygen species (ROS) in neurodegenerative diseases, and more recent data on posttranslational modifications such as S-nitrosylation, which can contribute to protein misfolding and the progression of neurodegenerative diseases. In the case of the proteindisulfide isomerase (PDI), S-nitrosylation inhibits the enzymatic activity leading to the accumulation of polyubiquitinated proteins. This activates the unfolded protein response, which contributes to neuronal cell injury and loss. The formation of Snitrosylated PDI occurs in several human diseases, including $A D$ and $P D$. Lipton then reviewed the molecular basis for the action of memantine in neurological diseases. These are mediated, at least in part, by overactivation of $N$-methyl-Daspartate (NMDA)-type glutamate receptors, producing excessive $\mathrm{Ca}^{2+}$ influx through the receptor's associated ion channel and consequent free-radical formation. Memantine, like the second generation-derivatives NitroMemantines, block excessive activation of NMDA receptors via a mechanism of uncompetitive open-channel block with a rapid 'off-rate'.
This mechanism affords blockade of the NMDA receptor during excessive activity without disrupting normal activity. In the case of Nitromemantines, the neuroprotective efficacy is enhanced by coupling $\mathrm{NO}$ to Memantine to target the thiol groups on the redox modulatory site(s) of the NMDA receptor. In contrast to PDI, S-nitrosylation of the NMDA receptor decreases its excessive activity and results in neuroprotection.

Philipe Gasque (Cardiff, UK and La Reunion, France) illustrated that several 'eat me' and 'not eat me' signals, which may be expressed by neurons in the brain together with their interacting partners on 'professional' or 'amateur' macrophages. In particular, a new family of receptors (CD93, CD141, and CD248) seem to have a relevant role in controlling the local innate immune response. An intriguing possibility raised by Gasque is that of 're-educating' and stimulating the resident phagocytes to promote clearance of pathogens, toxic cell debris and apoptotic/necrotic cells. Giovanbattista Bonanno (Genoa, Italy) reported on the $\mathrm{Ca}^{2+}$-dependent neurotransmitter release induced in gliosomes by high mobility group box 1 (HMGB1), a potent proinflammatory cytokine-like factor originally identified as a DNA-binding protein and a transcriptional regulator. As reported by Alberto Chiarugi (Florenz, Italy), HMGB1 maintains a nuclear localization in apoptotic cells, whereas in necrotic cells it is released into the extracellular space activating an autoamplifying inflammatory signalling cascade. In vitro and in vivo data seem to support an active role of HMGB1 during ischemia in the brain.

Andrea Volterra (Lausanne, Switzerland and Milan, Italy) discussed the current vision of astrocytes as an active component of the central nervous system (CNS). Calciumdependent glutamate release by astrocytes can be triggered by various stimuli, including the proinflammatory cytokine tumor necrosis factor- $\alpha$ (TNF- $\alpha$ ), acting via prostaglandins. In models of acute inflammation, the TNF- $\alpha$-dependent glutamate release by glia can become an important component

\footnotetext{
${ }^{1}$ Department of Pharmacobiology, University of Calabria, Cosenza, Italy; ${ }^{2}$ Centro di Neurobiologia Sperimentale Mondino-Tor Vergata, Rome, Italy; ${ }^{3}$ London Pain Consortium, University College London, London, UK; ${ }^{4}$ Department of Pharmacobiological Science, Faculty of Pharmacy, University Magna Graecia, Catanzaro, Italy; ${ }^{5}$ University Centre for Adaptive Disorders and Headache (UCHAD), University of Calabria, Cosenza, Italy and ${ }^{6}$ Burnham Institute for Medical Research, Del E. Webb Center for Neurosciences and Aging, La Jolla, CA, USA

*Corresponding author: G Bagetta, Department of Pharmacobiology, University of Calabria, via P. Bucci, Edificio Polifunzionale, Arcavacata di Rende, Cosenza 87036, Italy. Tel: + 39-0984-493462/3054; Fax: +39-0984-493462; E-mail: g_bagetta@ virgilio.it; published online 26.1.07
} 
of the degenerative process. Further on astrocytes, Milos Pekny (Goteborg, Sweden) discussed the role of these cells and the complement system in neuronal plasticity and the recent evidence that products from complement activation promote both basal- and ischemia-induced neurogenesis in the adult CNS. Particular emphasis was given to the data derived from mouse genetic models in which the absence of different intermediate filament (IF) proteins in astrocytes (e.g., $\mathrm{GFAP}^{-1-}$, $\mathrm{Vim}^{-/-}$, GFAP ${ }^{-1-} \mathrm{Vim}^{-/-}$) leads to attenuation of reactive gliosis with distinct pathophysiological and clinical consequences. The same mice subjected to several trauma models have also contributed to the understanding of the role of IF upregulation and reactive astrocytes in CNS trauma.

Matrix metalloproteinases (MMPs) were taken into picture by Anthony Turner's (Leeds, UK) overview on the different roles metalloproteinases can play in neurodegeneration and neuroprotection. Emphasis was given to metallopeptidases involved in the metabolism of the neurotoxic amyloid peptide such as neprilysins, insulin-degrading enzyme, angiotensinconverting enzyme, and plasmin. Data on a possible new role of MMPs in brain ischemia were also presented by Diana Amantea (Cosenza, Italy). MMP-2 and MMP-9 expression and activity increase during brain ischemia following middle cerebral artery occlusion. MMPs inhibitors, which prevent this increase, also reduce the infarct area and the processing of neuroinflammatory mediators such as interleukin (IL)-1 $\beta$.

Inflammatory and immune mechanisms in the periphery and the CNS also play an important role in chronic pain. Stephen Hunt (London, UK) highlighted the role of some of these inflammatory mediators such as TNF- $\alpha$ in the progression of different pain models.

Barbara Viviani (Milan, Italy) extended her previous findings to neurons exposed to the HIV-envelope glycoprotein gp120. Using a sandwich co-culture of primary hippocampal neurons and glia, she showed that IL-1 $\beta$ released from glia by gp120 contributes to neuronal death through tyrosine phosphorylation and trafficking of NMDA receptors.

Richard Ransohoff (Cleveland, USA) presented an overview of the complex world of chemokines and their receptors. In the CNS, constitutive chemokines support developmental and neurophysiological functions as well as regulating the activation of microglia. Because of their key role in both physiological and pathological conditions, chemokines and their receptors are attractive drug targets for the regulation of inflammation and immunity.

Diego Centonze (Rome, Italy) presented electrophysiological data on corticostriatal brain slices from mice with experimental autoimmune encephalomyelitis, a preclinical model of multiple sclerosis. In this system, striatal neurons show increased glutamatergic transmission and decreased GABAergic function suggesting that important processes of synaptic dysfunction occur during the early phases of the disease.
Tony Wyss-Coray (Stanford, USA) reviewed the evidence for immune and inflammatory pathways as modulators of AD. Mouse models with genetic manipulations of inflammatory pathways indicate that inflammatory processes may be a driving force of this pathology. However, a growing number of animal studies also support the idea that some inflammatory responses are beneficial and may be effective in preventing or treating disease. Joe Rogers (Sun City, USA) further analyzed the role of neuroinflammation in $A D$ before discussing more recent findings on neuroinflammatory mechanisms in the context of PD. Roger's data point towards $\mathrm{A} \beta$ deposition stimulating inflammatory responses, whereas neurons represent a bystander target in AD. In PD, dopamine itself may stimulate activated microglia to attack dopaminergic neurons, suggesting therefore that neurons are a direct, selective target in the disease. Also, in the context of PD, Howard Gendelman (Omaha, USA) reported on the 'protective and destructive role' of T lymphocytes and presented data on $\alpha$-synucleinlinked neuroimmunity in PD models. Innate immunity may contribute to the degeneration of the nigrostriatal dopaminergic pathway. Also, T lymphocytes present in brain tissue may affect disease progression. The immunization strategy with random amino-acid polymer copolymer-1 resulted in significant protection of nigrostriatal neurons from MPTP-induced neurodegeneration, and the effect was abrogated by depletion of donor T cells.

Luisa Minghetti's (Rome, Italy) talk focused on cyclooxygenase-2 (COX-2), a major player in inflammatory reactions. Data from prion animal models showed increased hippocampal levels of $\mathrm{PGE}_{2}$ and a strong induction of COX-2 expression in microglia associated with the progression of the disease. Elevated levels of $\mathrm{PGE}_{2}$ in the cerebrospinal fluid of patients affected by genetic or sporadic Creutzfeldt-Jacob disease support the animal studies.

Linda Van Eldik (Chicago, USA) illustrated a novel integrative drug discovery approach to identify new compounds targeted to modulate glial proinflammatory cytokine upregulation. In animal models of $A D$ and acute traumatic brain injury, the novel compound presented, Minozac, acts as a selective suppressor of excessive glial proinflammatory cytokine production and attenuates synaptic dysfunction and hippocampal-dependent behavioral deficits. This type of therapeutics could have a broad impact across diverse neurological disease indications.

Acknowledgements. The IXth Workshop on Apoptosis in Biology and Medicine (13-16 September 2006, Parghelia, VV, Italy) was organized with the financial support of the National Institute of Health (ISS, Rome, Italy), the University of Catanzaro 'Magna Graecia', Catanzaro (Italy), the ISN-CNR, Section of Catanzaro (Catanzaro, Italy), the Department of Pharmacobiology, University of Calabria, Arcavacata di Rende (CS, Italy), the International Society of Neurochemistry (ISN), the Italian Society of Pharmacology (SIF), and Elsevier, Inc. 